\section{>Imaging life with thin sheets of light}

The revival of light-sheet microscopy opens new possibilities for the imaging of living processes.

Optical sectioning microscopy techniques such as confocal or multiphoton microscopy have enabled three-dimensional imaging of living biological samples. But despite the many advances in this field, imaging strategies that offer high speeds, large fields of view or long-term imaging capacity are still in need to image whole cells, tissues and organisms at high resolution.

In the early 1900s, German scientists used a form of planar illumination to study colloidal solutions by scattered light imaging. Ninety years later, that work gave birth to a microscopy technique often called 'selective plane illumination microscopy' or 'fluorescence light-sheet microscopy'.

In this technique, the specimen is illuminated from the side with a thin laminar sheet of light and the emitted fluorescence is collected from above or below the sample.
Confining the excitation to a very thin two-dimensional area that is progressively moved through the specimen allows for an overall reduction of phototoxicity and photobleaching, fast imaging, high-sensitivity detection and three-dimensional optical sectioning.

Light-sheet microscopy can be done in single-photon or two-photon excitation modes, and the illumination strategy can be static and spread over the entire field of view or follow a line-scanning approach.

Recent years have seen great developments in this microscopy method and increased uptake thanks to the appearance of commercial products. Its application to the imaging of developmental processes in whole living organisms amazed the scientific community several years ago, and this year its potential has been extended in several ways, including to the imaging of single living cells. Eric Betzig and colleagues used Bessel beams-very narrow non-diffracting light rays each surrounded by concentric rings of less intense lightin combination with two-photon excitation or structured illumination to image

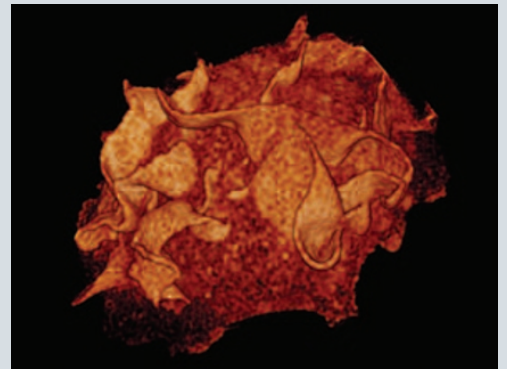

Membrane ruffles in a kidney cell line imaged using a variation of light-sheet microscopy. Image courtesy of Liang Gao.

subcellular structures in living cells at high resolution (Nat. Methods 8, 417-423; 2011). And an even better resolution is possible by combining plane illumination regimes with super-resolution microscopy (Nat. Methods 8, 1047-1049; 2011).

We expect to see many more improvements in imaging speed and resolution in the coming years. There is little question that a brilliant future lies ahead for the use of sheet-based illumination to study biological processes, enabling an in-depth view of the living - no matter how big or small.

Erika Pastrana

\section{\>Non-model organisms}

\section{Next-generation sequencing is} broadening the application of genetic and genomic studies to the panoply of life.

Model organisms satisfy a research need for genetic uniformity and live happily in the controlled hum of the laboratory. But existing models lack many interesting traits and are limited when it comes to answering evolutionary and ecological questions. Advances in sequencing have radically expanded the reach of genetic studies to non-model organisms and wild populations, making this an exciting area to watch.

Much of evolutionary and ecological research attempts to zero in on sequence variation that leads to functional phenotypic variation. The key to finding associations is to assess multiple markers in many individuals: what next-generation sequencing can do cheaply and often in one experiment. One class of genotyping-by-sequencing approach generates markers en masse without the need for a reference sequence. Restriction site-associated DNA sequencing and

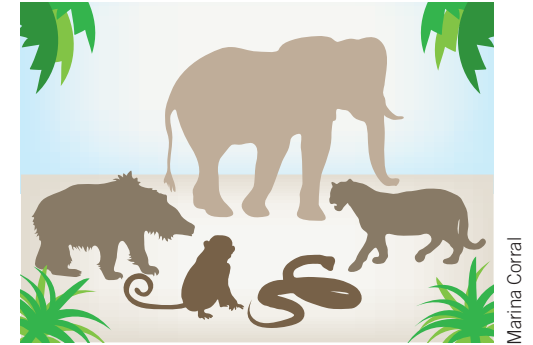

Genomic approaches open a jungle of possibilities.

complexity reduction of polymorphic sequences are examples that only sequence short stretches around a specific restriction site dispersed throughout the genome. Markers generated in this way can be used for quantitative trait locus mapping, examining phylogeography and tracking evolution in natural populations.

These approaches are revealing interesting insights into processes such as adaptive radiation - the evolution of phenotypic diversity within a lineage. Targeted or candidate gene sequencing can similarly be used to identify allelic variation driving processes such as mimicry in butterfly wings, a fascinating example of convergent evolution. Complementary de novo transcriptome-assembly tools and digitaltagging approaches allow studies of changes in coding sequences and gene expression in the absence of a reference sequence.

Plummeting costs and sophisticated bioinformatics are making whole-genome sequencing projects commonplace and enabling comparative genomic studies. Organisms with sequenced genomes in key phylogenetic positions can also be better exploited for developmental studies. For example, sequencing of spike moss, one of the earliest plants with true roots, can help to understand how these structures first evolved. Non-model organism methods benefit from having close relatives with wellannotated reference sequences. The promise of large-scale whole-genome sequencing projects such as the Genome $10 \mathrm{~K}$ Project is to anchor investigation of relatives in a diversity of phylogenetic neighborhoods, including many that can be helped by conservation genomic studies.

Population genomic comparisons will soon be possible, and we look forward to seeing the benefits of genomic science applied to interesting organisms that have yet to share the spotlight.

Tal Nawy 